\title{
THE QUALITY ASSESSMENT OF BOOKBINDING STRENGTH FOR POLYVINYL ACETATE ADHESIVE (PVAC) AND NANO-MODIFIED PVAC ADHESIVES
}

\author{
Gorana Petković (iD), Suzana Pasanec Preprotić (iD, Marina Vukoje (iD \\ University of Zagreb, Faculty of Graphic Arts, Zagreb, Croatia
}

\begin{abstract}
One of the most common and cost-effective book binding method is adhesive binding method suitable for medium volume books (50-250 pages). Depending on run volume and printing technology, different adhesive binding techniques can be used. In this research, for short run book production, doublefan binding was used, because it can achieve up to $20 \%$ higher binding strength compared to perfect binding technique. In addition, for adhesive binding book production, polyvinyl acetate dispersions (PVAc), hot-melts (HM) and polyurethane adhesives (PUR) can be used. Today, water-based PVAC adhesives are more environmentally acceptable and numerous studies dealing with modification of PVAc adhesives have been carried out, in order to achieve the comparable or higher quality of adhesive joints for selected application. The aim of this research is to improve the binding performance of PVAc adhesive by adding $1 \%$ of nanoparticles, specifically hydrophobic fumed silica $\left(\mathrm{SiO}_{2}\right)$ or hydrophilic fumed titanium dioxide $\left(\mathrm{TiO}_{2}\right)$ powder. ISO specifies a test method (ISO 19594) for the quality assessment of adhesive binding by pulling out a single sheet from a book block, page-pull test. Pulled sheets have to be evaluated visually and in a consistent manner, since during the procedure the sheet can be pulled out completely without significant damage, raptured roughly parallel to the binding edge, raptured not parallel to the binding edge or show significant adhesive penetration. In this research, the binding strength of 8 specimens, at 4 different test positions (10\%, 25\%, 50\% and $90 \%$ of the total number of pages), for 5 different groups of book blocks and 3 different types of PVAc adhesive (neat PVAc, PVAc $+1 \% \mathrm{SiO}_{2}, \mathrm{PVAc}+1 \% \mathrm{TiO}_{2}$ ) was evaluated. All the measurements were performed under the same conditions (volume, trimmed size, binding technique and conditions, drying and pressing time). For the evaluation of quality assessment of bookbinding strength, different paper types were used for each group - woodfree uncoated, woodfree coated, containing wood bulky, woodfree office and office paper containing recycled fibres. According to ISO 19594 standard, quality levels for the binding strength of adhesive bound products are not enough. It is also necessary to calculate the coefficient of variation in order to evaluate the consistency of binding quality. According to obtained results, visual evaluation of test sheets and book blocks opening behaviour, the best quality was achieved for book blocks with $P V A c+1 \% \mathrm{SiO}_{2}$ adhesive. Compared to a neat PVAc, the binding strength for all groups of book blocks was $10-26 \%$ higher while coefficient of variation was $43-71 \%$ lower. Results for PVAc $+1 \%$ $\mathrm{TiO}_{2}$ adhesive did not show any significant improvement or deterioration of the binding strength while coefficient of variation was notably lower, $4 \%-58 \%$, compared to neat PVAc.
\end{abstract}

Key words: binding strength, page-pull test, polyvinyl acetate adhesive, nano-modified adhesive

\section{INTRODUCTION}

According to European Federation for Print and Digital Communication volume of printed books will decline in favour of short runs, just-in-time, digitally printed books, together with an increased use of electronic publishing. Future business models have to meet new customer's requirements by switching to short run production, elimination of overstocking, allowing the customer to "sell first and print later", enabling versioning and personalization (Intergraf, 2015). All above described business models eliminate the need for storage and reduce publisher's profitable risk, but also may cause a reduction of delivery time and require constant and higher degree of the end product quality (Petković et al, 2017). Due to advancement in adhesive binding technology and shorter delivery time, adhesive binding is growing in popularity across all types of books and it is suitable for medium volume books (5-250 pages). The adhesive binding technique directly influence the quality of the end product. In perfect binding, the spine of the book block have to be mechanically pre-treated, in order to get higher roughness and enlargement of surface for adhesive application. In double-fan binding, book blocks are bending on both sides during adhesive application, allowing penetration of adhesive between leaves. The binding strength of double-fan binding is up to $20 \%$ higher with more uniform adhesive application (Petković et al, 2017). Currently used adhesives in bookbinding belong into three main categories: water-based emulsions (e.g. PVAc), hot melts (e.g. EVA) and reactive hot melts (e.g. PUR). Selection of adhesive type depends upon the nature of adherends, performance requirements, production volume and bonding processes. For the optimum performance, 
adhesive should have excellent flexibility, toughness (without stiffness) and fatigue resistance. In addition, adhesive should have the ability of application in low-cost, high-speed processes and it should be able to survive storage conditions and repetitive stress (Petrie, 2008). Today, water-based adhesives are considerably more environmentally, they have the highest market share and they are mainly used in the paper industry (46.3\%) (Jović et al, 2011). Water-based polyvinyl acetate adhesive (PVAc) has many desirable features and advantages, with only few limitations, as presented in Table 1.

Table 1: Main advantages and limitations of PVAc adhesive

\begin{tabular}{|c|c|c|}
\hline & FEATURE & REFERENCE \\
\hline \multirow{7}{*}{$\begin{array}{l}\text { 岁 } \\
\text { 峞 } \\
\text { 峞 }\end{array}$} & $\begin{array}{l}\text { easy and wide for application } \\
\text { (paper, plastic, metal foil, leather, cloth, wood, etc.) }\end{array}$ & (Paris, 2000; Šedivka et al, 2015) \\
\hline & low cost and availability & (Ebnesajjad, 2008; Paris, 2000; Thomson, 2014) \\
\hline & no toxicity problems & (Packham, 2005; Paris, 2000; Thomson, 2014) \\
\hline & no aging problems & (Rebsamen, 1983) \\
\hline & dried film holds high joint flexibility & (Thomson, 2014) \\
\hline & does not damage the equipment & (Thomson, 2014) \\
\hline & provides invisible, colourless and transparent glue lines & (Ebnesajjad, 2008; Salvini et al, 2009; Thomson, 2014) \\
\hline \multirow{4}{*}{ 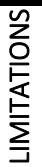 } & low resistance to weather and moisture & (Ebnesajjad, 2008; Salvini et al, 2009) \\
\hline & poor resistance to most solvents & (Ebnesajjad, 2008; Salvini et al, 2009) \\
\hline & slow setting speed & (Packham, 2005) \\
\hline & curing for 1-7 days is recommended before handling & (Ebnesajjad, 2008) \\
\hline
\end{tabular}

Besides binding technique and adhesive selection, the proper paper selection is crucial to the end product quality as well. Compatible materials selection provides the best performance properties of book products, including page-pull, lay-flat and easy-open flexible spine characteristics (Petrie, 2008). The number of possible substrates, coatings and end uses are seemingly limitless, but 92\% of all printed books in Europe are printed on woodfree paper (Intergraf, 2017).

Various studies have been focused on improving the properties of PVAc adhesives through their modification, especially by nanoparticles addition (nano clay, cellulose nanofibrils, silica or titanium dioxide) (Bardak et al, 2016; Wen et al, 2008; Aydemir et al, 2016; Laskowska et al, 2017). Properties of different conventional materials can be changed by nanoparticles addition while their small amount can cause significant changes in properties (Ramljak, 2017). In addition, these modifications can cause some side effects, such as a lower shelf-life, yellowing of adhesive or reduction of their performance (Tanket et al, 2016; Bonnefond et al, 2013). The adhesive industry continues to develop, and it is focus on improvements in drying mechanisms, bond strength, flexibility and application versatility.

\section{MATERIALS AND METHODS}

\subsection{Materials}

\subsubsection{Paper}

For a book production five types of paper were used as listed in Table 2. All of them belong to a different paper type group, based on fibre composition.

Table 2: List of used papers

\begin{tabular}{cccc}
\hline TRADE NAME & TYPE & GRAMMAGE $\left[\mathrm{g} / \mathrm{m}^{2}\right]$ & ABBREVIATION \\
\hline Amber Graphics & woodfree uncoated & 100 & WFU \\
\hline Garda Gloss & woodfree coated & 115 & WFC \\
\hline Munken White & containing wood (bulky) & 90 & CW \\
\hline Navigator Universal & woodfree, office & 80 & WF office \\
\hline Recy Office & containing recycled (100\%), office & 80 & CR office \\
\hline
\end{tabular}

\subsubsection{Adhesive}

Used polyvinyl acetate adhesive (Signokol L) is water dispersion of vinyl acetate homopolymers with polyvinyl alcohol and addition of plasticizers. According to material safety data sheet, it has $45 \pm 2 \%$ of solid 
content and the varying amount of water (0 - 5\%) can be added. Preliminary study showed that the best adhesion was achieved with 0 - 2.5\% of water (Petković et al, 2016) and thus in this research, no water was added in order to eliminate water as an influential factor.

\subsubsection{Nanoparticles}

For PVAc adhesive modification, hydrophobic fumed silica ( $\mathrm{SiO}_{2}$, AEROSIL R 8200) and hydrophilic fumed titanium dioxide $\left(\mathrm{TiO}_{2}\right.$, AEROXIDE P25) were used. Both are odourless, solid, white powders with approximately same tamped density $(140 \mathrm{~g} / \mathrm{L})$, but a different BET surface area $\left(135-185 \mathrm{~m}^{2} / \mathrm{g}-\mathrm{SiO}_{2} ; 35\right.$ $\left.-65 \mathrm{~m}^{2} / \mathrm{g}-\mathrm{TiO}_{2}\right)$ and assay based on ignited material $\left(\mathrm{SiO}_{2} \geq 99.8 \% ; \mathrm{TiO}_{2} \geq 99.5 \%\right)$.

\subsection{Methods}

\subsubsection{Adhesive preparation}

Three types of adhesives were used - neat PVAc adhesive and PVAc adhesive modified with $1 \%$ of $\mathrm{SiO}_{2}$ and $1 \%$ of $\mathrm{TiO}_{2}$ nanoparticles. After adding $1 \%$ of nanoparticles to neat PVAc adhesive, IKA T 25 digital ULTRATURRAX disperser was used for mixing and adhesive homogenisation. Adhesive was stirred for 15 minutes, firstly 5 minutes at lower stirring speed (from 3500 to $7000 \mathrm{rpm}$ ) and then 10 minutes at $7000 \mathrm{rpm}$. The same mixing procedure was used for both nano-modified adhesives.

\subsubsection{Specimens preparation}

For the determination of binding strength, 120 book blocks were made ( 24 for each paper type). According to ISO 19594 standard guidelines, at least five specimens for each test should be used. In this research, 8 specimens for each test were measured. All book blocks were unprinted, without covers, with volume of 100 pages and $165 \times 240 \mathrm{~mm}$ in trimmed size. Binding units were individual leaves, bonded with doublefan binding technique, pressed for 48 and dried for 72 hours. Spine width was 5-7 mm, depending on paper type and grammage. Binding was performed at room temperature of $20 \pm 5{ }^{\circ} \mathrm{C}$ and relative humidity of 35 $-75 \%$.

\subsubsection{Determination of the tensile index $(T)$}

Tensile index (T) was determined according to standard method (ISO 1924-1). Measurements were performed on Enrico Toniolo Dynamometer Tensomini Super machine. Tensile index can be calculated from paper tensile strength according to Equation 1.

$$
T=\frac{F}{g}\left[\frac{N m}{g}\right]
$$

Where: $\mathrm{F}$ - tensile strength, $\mathrm{g}$ - paper grammage.

\subsubsection{Determination of the binding strength (BS)}

According to ISO 19594, the binding strength is evaluated by pulling out a single sheet from the book block and measuring the maximum force resulting from the page-pull procedure. Page-pull test was performed according ISO 187, standardized conditioning and testing atmosphere, on IDM Page Pull Tester (model P0011). In addition to measurement of the maximum force, it is necessary to inspect all tested sheets, record their states and classify them in one of four categories listed in Table 3. Tested sheets marked with D should be excluded from any calculation. Tested sheets marked with B or C should be marked with ">" and excluded from the calculation of the coefficient of variation.

Table 3: Test sheet state classification

\begin{tabular}{|c|c|c|}
\hline \multirow{4}{*}{ 晨岕怠 } & A & pulled out completely without significant damage \\
\hline & B & ruptured roughly parallel to the binding edge \\
\hline & C & with the rupture extending to the binding edge \\
\hline & D & with significant glue penetration \\
\hline
\end{tabular}

The binding strength (BS) of the specimen is calculated according to ISO 19594:2017 and Equation 2. The quality levels for the evaluation of obtained binding strength results are listed in Table 4. 


$$
B S=\frac{F_{\max }}{l}\left[\frac{N}{\mathrm{~cm}}\right]
$$

Where: F - maximum force, I - spine length.

Table 4: The ISO 19594 quality levels for the evaluation of binding strength (QL $\left.L_{B S}\right)$

\begin{tabular}{cc}
\hline QUALITY LEVEL (QLBS) & $\begin{array}{c}\text { BINDING STRENGTH } \\
{[\mathrm{N} / \mathrm{cm}]}\end{array}$ \\
\hline very good durability & $>7.0$ \\
\hline good durability & $\leq 7.0 ;>6.1$ \\
\hline sufficient durability & $\leq 6.1 ;>5.1$ \\
\hline poor durability & $\leq 5.1$ \\
\hline
\end{tabular}

The quality levels for the consistency of binding quality, based on coefficient of variation (CV), are listed in Table 5.

Table 5: The ISO 19594 quality levels for the consistency of the binding quality (QLCV)

\begin{tabular}{cc}
\hline QUALITY LEVEL (QLCV) & COEFFICIENT OF VARIATION \\
\hline very good consistency & $\leq 0.10$ \\
\hline good consistency & $>0.10 ; \leq 0.15$ \\
\hline sufficient consistency & $>0.15 ; \leq 0.20$ \\
\hline poor consistency & $>0.20$ \\
\hline
\end{tabular}

\subsubsection{Determination of drape factor (DF)}

Drape factor (DF) of paper is the main factor influencing the book block opening behaviour (drapeability). It represent single unglued sheet of paper and indicates how much leverage a paper is capable of exerting at the glue line (Jermann, 2008). After measurement of paper extend length, until it drops one inch at the leading edge, drape factor was evaluated according to Equation 3.

$$
D F=\frac{l}{2.54} \times 10
$$

Where I represents extend length of paper.

\section{RESULTS}

\subsection{Paper tensile index}

Calculated tensile index values, for machine (MD) and cross (CD) directions are presented in Table 6.

Table 6: Paper tensile indexes

\begin{tabular}{ccc}
\hline PAPER & $\begin{array}{c}\text { TENSILE INDEX (MD) } \\
{[\mathrm{Nm} / \mathrm{g}]}\end{array}$ & $\begin{array}{c}\text { TENSILE INDEX (CD) } \\
{[\mathrm{Nm} / \mathrm{g}]}\end{array}$ \\
\hline WFU & 47.09 & 23.29 \\
\hline WFC & 31.66 & 18.41 \\
\hline CW & 45.68 & 16.96 \\
\hline WF office & 76.03 & 28.51 \\
\hline CR office & 41.15 & 14.03 \\
\hline
\end{tabular}

\subsection{Binding strength quality and binding quality consistency}

Every book block specimen, with spine thickness of at least $10 \mathrm{~mm}$, should be tested at minimum three test positions ( $10 \%, 50 \%$ and $90 \%$ of total number of pages). Book blocks with thickness less than $10 \mathrm{~mm}$ can be tested only at one test position. During page-pull procedure, 8 specimens for every adhesive and paper type were measured at four different test positions - 10\%,25\%, $50 \%$ and $90 \%$ (binding unit: 5, 13, 25 and 45). All binding strength results are listed in Tables $7-11$, with the state of tested sheet classification 
(A-D), total mean values, standard deviation and coefficient of variation. Considering the obtained results and quality levels listed in Tables 4 and 5, description of a binding strength and binding quality consistency is presented. Considering the mean values for binding strength and coefficient of variation of neat PVAc as reference value, percentage for durability and consistency improvement (e.g. binding strength enhancement and coefficient of variation reduction) is calculated and presented in the two last rows of each table.

Table 7: Binding strength (BS) and coefficient of variation values (CV), for WFU book blocks, with binding (QL $\left.L_{B S}\right)$ and consistency $\left(Q L_{C V}\right)$ quality levels. Durability and consistency improvement of nano-modified adhesives compared with a neat PVAc [\%].

\begin{tabular}{|c|c|c|c|c|c|c|c|c|c|c|c|c|c|c|c|c|c|c|c|c|c|c|c|c|}
\hline \multicolumn{25}{|c|}{$W F U-100 \mathrm{~g} / \mathrm{m}^{2}[\mathrm{~N} / \mathrm{cm}]$} \\
\hline & \multicolumn{8}{|c|}{ PVAc (reference) } & \multicolumn{8}{|c|}{$\mathrm{PVAc}+1 \% \mathrm{SiO}_{2}$} & \multicolumn{8}{|c|}{$\mathrm{PVAc}+1 \% \mathrm{TiO}_{2}$} \\
\hline & \multicolumn{2}{|c|}{$10 \%$} & \multicolumn{2}{|c|}{$25 \%$} & \multicolumn{2}{|c|}{$50 \%$} & \multicolumn{2}{|c|}{$90 \%$} & \multicolumn{2}{|c|}{$10 \%$} & \multicolumn{2}{|c|}{$25 \%$} & \multicolumn{2}{|c|}{$50 \%$} & \multicolumn{2}{|c|}{$90 \%$} & \multicolumn{2}{|c|}{$10 \%$} & \multicolumn{2}{|c|}{$25 \%$} & \multicolumn{2}{|c|}{$50 \%$} & \multicolumn{2}{|c|}{$90 \%$} \\
\hline 1 & 9.89 & A & 5.64 & $B$ & 9.44 & A & 18.3 & $\mathrm{D}$ & 9.14 & $\mathrm{~A}$ & 9.46 & $\mathrm{~A}$ & 16.3 & $\mathrm{D}$ & 10.0 & $A$ & 8.42 & $A$ & 8.73 & A & 12.4 & $\mathrm{D}$ & 8.33 & $A$ \\
\hline 2 & 7.85 & A & 9.44 & B & 9.39 & $\mathrm{~A}$ & 9.8 & c & 10.0 & $\mathrm{~A}$ & 14.4 & $\mathrm{D}$ & 9.71 & A & 9.35 & $\mathrm{~A}$ & 8.67 & $\mathrm{~A}$ & 8.61 & A & 12.5 & $\mathrm{D}$ & 9.27 & A \\
\hline 3 & 8.46 & A & 13.9 & $\mathrm{D}$ & 8.39 & A & 18.2 & $\mathrm{D}$ & 10.4 & $\mathrm{~A}$ & 9.14 & $B$ & 9.02 & C & 9.69 & $\mathrm{~A}$ & 9.10 & A & 15.1 & $\mathrm{D}$ & 9.21 & $\mathrm{~A}$ & 8.08 & A \\
\hline 4 & 9.96 & c & 9.71 & $\mathrm{~A}$ & 9.79 & $\mathrm{~B}$ & 9.64 & $\mathrm{~A}$ & 9.89 & A & 10.2 & $\mathrm{~B}$ & 10.3 & c & 9.37 & $\mathrm{~A}$ & 10.1 & $\mathrm{D}$ & 9.10 & $\mathrm{~A}$ & 15.8 & $\mathrm{D}$ & 7.25 & $A$ \\
\hline 5 & 9.60 & $A$ & 8.83 & $\mathrm{~B}$ & 9.50 & $\mathrm{~A}$ & 16.8 & $\mathrm{D}$ & 10.1 & $\mathrm{~A}$ & 14.4 & $\mathrm{D}$ & 14.7 & $\mathrm{D}$ & 9.52 & $\mathrm{~A}$ & 8.87 & A & 9.14 & $\mathrm{~A}$ & 8.83 & $B$ & 7.66 & $A$ \\
\hline 6 & 9.96 & $\mathrm{~A}$ & 9.94 & $B$ & 4.79 & $\mathrm{~B}$ & 9.27 & $\mathrm{~A}$ & 8.81 & $\mathrm{~A}$ & 9.52 & c & 9.14 & A & 9.42 & $\mathrm{~A}$ & 9.35 & $\mathrm{~A}$ & 9.46 & $\mathrm{~A}$ & 6.81 & $\mathrm{~A}$ & 9.50 & $A$ \\
\hline 7 & 9.71 & $\mathrm{~A}$ & 18.3 & $\mathrm{D}$ & 8.54 & $\mathrm{~A}$ & 16.3 & $\mathrm{D}$ & 9.39 & $\mathrm{~A}$ & 9.48 & A & 9.62 & $\mathrm{~A}$ & 9.08 & $\mathrm{~A}$ & 9.00 & $B$ & 6.75 & $\mathrm{~A}$ & 15.3 & $\mathrm{D}$ & 7.42 & $\mathrm{~A}$ \\
\hline 8 & 8.29 & $\mathrm{~A}$ & 6.04 & $\mathrm{~A}$ & 5.04 & $\mathrm{~B}$ & 8.42 & c & 16.3 & $\mathrm{D}$ & 9.25 & $\mathrm{~A}$ & 14.2 & $\mathrm{D}$ & 9.39 & $\mathrm{~A}$ & 8.87 & $\mathrm{~A}$ & 14.4 & $\mathrm{D}$ & 6.66 & $\mathrm{~A}$ & 8.75 & $A$ \\
\hline$\overline{\mathrm{x}}$ & \multicolumn{8}{|c|}{8.669} & \multicolumn{8}{|c|}{9.562} & \multicolumn{8}{|c|}{8.437} \\
\hline$\sigma$ & \multicolumn{8}{|c|}{0.988} & \multicolumn{8}{|c|}{0.379} & \multicolumn{8}{|c|}{0.888} \\
\hline BS & \multicolumn{8}{|c|}{$>8.7$} & \multicolumn{8}{|c|}{$>9.6$} & \multicolumn{8}{|c|}{$>8.4$} \\
\hline$\underset{(B S)}{Q \mathrm{QL}}$ & \multicolumn{8}{|c|}{ very good durability } & \multicolumn{8}{|c|}{ very good durability } & & & very gc & od c & durabi & & & \\
\hline $\mathrm{CV}$ & & & & 0.10 & & & & & & & & 0.03 & & & & & & & & 0.10 & & & & \\
\hline $\begin{array}{l}\mathrm{QL} \\
(\mathrm{CV})\end{array}$ & & & good & con & sistenc & & & & & & ery goc & od co & onsiste & ency & & & & & good & cons & sisten & & & \\
\hline & bility & in & orovem & ent & (BS ent & anc & cement & & & & & 10.3 & $30 \%$ & & & & & & & 2.68 & $8 \%$ & & & \\
\hline & onsist & $n c y$ & impro & vem & ent $(\mathrm{Cl}$ & red & duction & & & & & 63.8 & $9 \%$ & & & & & & & 4.26 & $6 \%$ & & & \\
\hline
\end{tabular}

Table 8: Binding strength (BS) and coefficient of variation values (CV), for WFC book blocks, with binding $\left(Q L_{B S}\right)$ and consistency $\left(Q L_{C V}\right)$ quality levels. Durability and consistency improvement of nano-modified adhesives compared with a neat PVAc [\%].

\begin{tabular}{|c|c|c|c|c|c|c|c|c|c|c|c|c|c|c|c|c|c|c|c|c|c|c|c|c|}
\hline \multicolumn{25}{|c|}{$W F C-115 \mathrm{~g} / \mathrm{m}^{2}[\mathrm{~N} / \mathrm{cm}]$} \\
\hline & \multicolumn{8}{|c|}{ PVAc (reference) } & \multicolumn{8}{|c|}{$\mathrm{PVAC}+1 \% \mathrm{SiO}_{2}$} & \multicolumn{8}{|c|}{$\mathrm{PVAc}+1 \% \mathrm{TiO}_{2}$} \\
\hline & \multicolumn{2}{|c|}{$10 \%$} & \multicolumn{2}{|c|}{$25 \%$} & \multicolumn{2}{|c|}{$50 \%$} & \multicolumn{2}{|c|}{$90 \%$} & \multicolumn{2}{|c|}{$10 \%$} & \multicolumn{2}{|c|}{$25 \%$} & \multicolumn{2}{|c|}{$50 \%$} & \multicolumn{2}{|c|}{$90 \%$} & \multicolumn{2}{|c|}{$10 \%$} & \multicolumn{2}{|c|}{$25 \%$} & \multicolumn{2}{|c|}{$50 \%$} & \multicolumn{2}{|c|}{$90 \%$} \\
\hline 1 & 3.08 & $\mathrm{~A}$ & 3.81 & $\mathrm{~A}$ & 2.10 & A & 7.69 & $A$ & 5.98 & $\mathrm{~A}$ & 3.73 & $A$ & 3.25 & A & 4.02 & $A$ & 2.62 & A & 2.25 & $\mathrm{~A}$ & 5.73 & $\mathrm{~A}$ & 8.83 & $\mathrm{~A}$ \\
\hline 2 & 5.42 & $A$ & 2.44 & $A$ & 2.54 & $\mathrm{~A}$ & 3.29 & A & 6.69 & A & 6.04 & $\mathrm{~A}$ & 4.56 & $\mathrm{~A}$ & 8.54 & A & 5.75 & $A$ & 3.79 & $\mathrm{~A}$ & 2.96 & $A$ & 2.83 & $\mathrm{~A}$ \\
\hline 3 & 2.25 & $\mathrm{~A}$ & 3.80 & $\mathrm{~A}$ & 2.60 & $\mathrm{~A}$ & 8.50 & $A$ & 4.85 & $\mathrm{~A}$ & 4.27 & $\mathrm{~A}$ & 3.35 & $\mathrm{~A}$ & 6.02 & $A$ & 2.87 & $\mathrm{~A}$ & 4.85 & $\mathrm{~A}$ & 3.54 & $A$ & 2.12 & $\mathrm{~A}$ \\
\hline 4 & 2.17 & $A$ & 2.77 & $A$ & 2.91 & $\mathrm{~A}$ & 6.44 & A & 5.39 & A & 5.98 & A & 3.46 & A & 4.69 & A & 4.58 & $\mathrm{~A}$ & 2.29 & A & 2.60 & $A$ & 3.33 & $A$ \\
\hline 5 & 3.92 & $A$ & 2.69 & $A$ & 4.98 & $\mathrm{~A}$ & 3.62 & $A$ & 5.48 & $A$ & 3.48 & $A$ & 4.00 & $A$ & 5.64 & $A$ & 2.79 & $A$ & 3.35 & $\mathrm{~A}$ & 5.31 & $A$ & 3.52 & $A$ \\
\hline 6 & 5.75 & $A$ & 5.19 & $\mathrm{~A}$ & 3.31 & $\mathrm{~A}$ & 5.79 & A & 4.60 & A & 3.23 & A & 3.92 & A & 4.67 & A & 2.29 & $\mathrm{~A}$ & 4.75 & $\mathrm{~A}$ & 2.64 & $A$ & 2.46 & $A$ \\
\hline 7 & 3.68 & $\mathrm{~A}$ & 2.50 & $\mathrm{~A}$ & 2.19 & $\mathrm{~A}$ & 7.58 & A & 6.12 & $\mathrm{~A}$ & 4.08 & $\mathrm{~A}$ & 6.52 & A & 5.10 & A & 2.37 & $\mathrm{~A}$ & 2.35 & A & 2.04 & $\mathrm{~A}$ & 4.83 & $\mathrm{~A}$ \\
\hline 8 & 3.21 & $A$ & 7.21 & $\mathrm{~A}$ & 2.67 & $\mathrm{~A}$ & 3.39 & A & 6.69 & $\mathrm{~A}$ & 6.79 & $\mathrm{~A}$ & 6.87 & $A$ & 5.95 & A & 3.62 & A & 2.96 & $\mathrm{~A}$ & 5.44 & A & 5.46 & $\mathrm{~A}$ \\
\hline$\overline{\mathrm{x}}$ & \multicolumn{8}{|c|}{4.047} & \multicolumn{8}{|c|}{5.125} & \multicolumn{8}{|c|}{3.662} \\
\hline$\sigma$ & \multicolumn{8}{|c|}{1.810} & \multicolumn{8}{|c|}{1.300} & \multicolumn{8}{|c|}{1.494} \\
\hline BS & \multicolumn{8}{|c|}{4.0} & \multicolumn{8}{|c|}{5.1} & \multicolumn{8}{|c|}{3.7} \\
\hline$\underset{(B S)}{\mathrm{QL}}$ & \multicolumn{8}{|c|}{ poor durability } & \multicolumn{8}{|c|}{ sufficient durability } & & & poo & $\mathrm{rdu}$ & rabilit & & & \\
\hline $\mathrm{CV}$ & & & & 0.4 & & & & & & & & 0.25 & & & & & & & & 0.4 & & & & \\
\hline $\begin{array}{l}\mathrm{QL} \\
\text { (cv) }\end{array}$ & & & poor & con & sisten & & & & & & poor & cons & sisten & & & & & & poor & con & sisten & & & \\
\hline & rability & $\min$ & rovem & ent & BS en & hanc & emen & & & & & 26.6 & $4 \%$ & & & & & & & 9.5 & $0 \%$ & & & \\
\hline & onsist & ncy & impro & vem & ent $(C$ & rea & ductio & & & & & 43.2 & $8 \%$ & & & & & & & 8.8 & $3 \%$ & & & \\
\hline
\end{tabular}


Table 9: Binding strength (BS) and coefficient of variation values (CV), for CW book blocks, with binding (QL $\left.L_{B S}\right)$ and consistency $\left(Q L_{C V}\right)$ quality levels. Durability and consistency improvement of nano-modified adhesives compared with a neat PVAC [\%].

\begin{tabular}{|c|c|c|c|c|c|c|c|c|c|c|c|c|c|c|c|c|c|c|c|c|c|c|c|c|}
\hline \multicolumn{25}{|c|}{$\mathrm{CW}-90 \mathrm{~g} / \mathrm{m}^{2}[\mathrm{~N} / \mathrm{cm}]$} \\
\hline & \multicolumn{8}{|c|}{ PVAc (reference) } & \multicolumn{8}{|c|}{$\mathrm{PVAC}+1 \% \mathrm{SiO}_{2}$} & \multicolumn{8}{|c|}{$\mathrm{PVAc}+1 \% \mathrm{TiO}_{2}$} \\
\hline & \multicolumn{2}{|c|}{$10 \%$} & \multicolumn{2}{|c|}{$25 \%$} & \multicolumn{2}{|c|}{$50 \%$} & \multicolumn{2}{|c|}{$90 \%$} & \multicolumn{2}{|c|}{$10 \%$} & \multicolumn{2}{|c|}{$25 \%$} & \multicolumn{2}{|c|}{$50 \%$} & \multicolumn{2}{|c|}{$90 \%$} & \multicolumn{2}{|c|}{$10 \%$} & \multicolumn{2}{|c|}{$25 \%$} & \multicolumn{2}{|c|}{$50 \%$} & \multicolumn{2}{|c|}{$90 \%$} \\
\hline 1 & 11.5 & $\mathrm{D}$ & 8.31 & $\mathrm{~A}$ & 13.7 & $\mathrm{D}$ & 9.69 & C & 10.2 & $\mathrm{~A}$ & 17.7 & $\mathrm{D}$ & 10.9 & $\mathrm{~A}$ & 10.6 & A & 9.96 & B & 8.29 & $\mathrm{~A}$ & 15.8 & $\mathrm{D}$ & 8.94 & A \\
\hline 2 & 4.23 & $\mathrm{~A}$ & 9.67 & $\mathrm{~A}$ & 8.94 & $\mathrm{~A}$ & 9.81 & $\mathrm{~A}$ & 9.81 & $\mathrm{~A}_{\mathrm{A}}$ & 10.1 & $\mathrm{~A}$ & 9.31 & $A$ & 13.3 & $\mathrm{D}$ & 15.4 & $\mathrm{D}$ & 16.7 & $\mathrm{D}$ & 10.6 & $\mathrm{D}$ & 8.94 & $A$ \\
\hline 3 & 9.75 & $B$ & 9.06 & $\mathrm{~A}$ & 5.60 & $B$ & 9.81 & $A$ & 9.56 & C & 9.23 & A & 9.79 & $A$ & 17.6 & $\mathrm{D}$ & 15.4 & $\mathrm{D}$ & 13.5 & $\mathrm{D}$ & 9.89 & $\mathrm{~A}$ & 7.58 & $B$ \\
\hline 4 & 9.25 & $A$ & 9.81 & A & 7.71 & C & 9.85 & $\mathrm{~A}$ & 10.1 & A & 16.6 & $\mathrm{D}$ & 9.89 & A & 9.39 & $A$ & 9.35 & $\mathrm{~A}$ & 9.31 & $\mathrm{~A}$ & 14.7 & $\mathrm{D}$ & 7.04 & $A$ \\
\hline 5 & 20.0 & $\mathrm{D}$ & 9.92 & A & 14.7 & $\mathrm{D}$ & 9.58 & $\mathrm{~A}$ & 9.75 & $\mathrm{~A}$ & 9.58 & $A$ & 10.7 & $A$ & 18.2 & $D$ & 9.44 & $\mathrm{~A}$ & 13.4 & $\mathrm{D}$ & 9.71 & $A$ & 7.25 & $A$ \\
\hline 6 & 8.60 & $\mathrm{C}$ & 9.58 & $\mathrm{~A}$ & 8.10 & $\mathrm{~A}$ & 15.1 & $\mathrm{D}$ & 9.33 & $\mathrm{~B}$ & 17.5 & $\mathrm{D}$ & 9.67 & A & 10.2 & $B$ & 7.67 & $\mathrm{~A}$ & 14.3 & $\mathrm{D}$ & 7.83 & $\mathrm{~B}$ & 9.38 & $A$ \\
\hline 7 & 14.9 & $\mathrm{D}$ & 14.9 & $\mathrm{D}$ & 9.50 & $\mathrm{~B}$ & 13.2 & $\mathrm{D}$ & 10.1 & $\mathrm{~A}_{\mathrm{A}}$ & 10.2 & $B$ & 10.1 & $\mathrm{~A}$ & 9.23 & $B$ & 9.44 & $\mathrm{~A}$ & 7.31 & $\mathrm{~A}$ & 8.10 & $\mathrm{~A}$ & 7.77 & $B$ \\
\hline 8 & 7.92 & $B$ & 9.83 & $A$ & 8.37 & C & 9.17 & $\mathrm{~B}$ & 9.33 & A & 9.08 & A & 9.56 & $\mathrm{~A}$ & 9.31 & $\mathrm{~A}$ & 9.04 & $A$ & 16.3 & $\mathrm{D}$ & 9.39 & $\mathrm{~A}$ & 8.85 & A \\
\hline $\bar{x}$ & \multicolumn{8}{|c|}{8.837} & \multicolumn{8}{|c|}{9.828} & \multicolumn{8}{|c|}{8.659} \\
\hline$\sigma$ & \multicolumn{8}{|c|}{1.434} & \multicolumn{8}{|c|}{0.500} & \multicolumn{8}{|c|}{0.878} \\
\hline BS & \multicolumn{8}{|c|}{$>8.8$} & \multicolumn{8}{|c|}{$>9.8$} & \multicolumn{8}{|c|}{$>8.6$} \\
\hline$\underset{(B S)}{Q}$ & \multicolumn{8}{|c|}{ very good durability } & \multicolumn{8}{|c|}{ very good durability } & & & very $g$ & ood c & durab & & & \\
\hline $\mathrm{CV}$ & & & & 0.1 & & & & & & & & 0.05 & & & & & & & & 0.10 & & & & \\
\hline $\begin{array}{l}\mathrm{QL} \\
(\mathrm{CV})\end{array}$ & & & sufficie & t $\mathrm{cc}$ & nsiste & ncy & & & & & ery goc & od co & onsist & ency & & & & & good & cons & sisten & & & \\
\hline & ability & $i m p$ & orovem & ent & BS ent & hane & emen & & & & & 11.2 & $21 \%$ & & & & & & & 2.0 & $1 \%$ & & & \\
\hline & onsist & $n c y$ & impro & vem & ent $(C)$ & rec & ductio & & & & & 68.1 & $3 \%$ & & & & & & & 37.0 & $11 \%$ & & & \\
\hline
\end{tabular}

Table 10: Binding strength (BS) and coefficient of variation values (CV), for WF office book blocks, with binding (QLBS) and consistency $\left(Q L_{C V}\right)$ quality levels. Durability and consistency improvement of nano-modified adhesives compared with a neat PVAC [\%].

\begin{tabular}{|c|c|c|c|c|c|c|c|c|c|c|c|c|c|c|c|c|c|c|c|c|c|c|c|c|}
\hline \multicolumn{25}{|c|}{ WF office $-80 \mathrm{~g} / \mathrm{m}^{2}[\mathrm{~N} / \mathrm{cm}]$} \\
\hline & \multicolumn{8}{|c|}{ PVAc (reference) } & \multicolumn{8}{|c|}{$\mathrm{PVAc}+1 \% \mathrm{SiO}_{2}$} & \multicolumn{8}{|c|}{$\mathrm{PVAc}+1 \% \mathrm{TiO}_{2}$} \\
\hline & \multicolumn{2}{|c|}{$10 \%$} & \multicolumn{2}{|c|}{$25 \%$} & \multicolumn{2}{|c|}{$50 \%$} & \multicolumn{2}{|c|}{$90 \%$} & \multicolumn{2}{|c|}{$10 \%$} & \multicolumn{2}{|c|}{$25 \%$} & \multicolumn{2}{|c|}{$50 \%$} & \multicolumn{2}{|c|}{$90 \%$} & \multicolumn{2}{|c|}{$10 \%$} & \multicolumn{2}{|c|}{$25 \%$} & \multicolumn{2}{|c|}{$50 \%$} & \multicolumn{2}{|c|}{$90 \%$} \\
\hline 1 & 3.96 & $\mathrm{~A}$ & 9.04 & $\mathrm{~A}$ & 4.08 & $\mathrm{~A}$ & 15.6 & $\mathrm{D}$ & 9.87 & $A$ & 8.96 & $\mathrm{C}$ & 15.7 & $\mathrm{D}$ & 8.73 & A & 8.81 & $A$ & 8.62 & $\mathrm{~A}$ & 9.21 & $A$ & 8.12 & $A$ \\
\hline 2 & 9.29 & A & 9.56 & $A$ & 8.89 & $\mathrm{~A}$ & 7.94 & $B$ & 8.77 & $\mathrm{~A}$ & 10.3 & $\mathrm{~A}$ & 8.75 & $c$ & 15.2 & $D$ & 9.25 & $B$ & 8.81 & $\mathrm{~A}$ & 8.21 & $\mathrm{~A}$ & 8.12 & A \\
\hline 3 & 5.64 & A & 9.44 & A & 15.2 & $\mathrm{D}$ & 9.04 & c & 8.85 & $\mathrm{~B}$ & 10.3 & $A$ & 9.48 & $A$ & 8.67 & $\mathrm{~B}$ & 8.60 & A & 9.17 & A & 9.14 & $A$ & 9.08 & A \\
\hline 4 & 9.64 & A & 6.42 & A & 8.92 & $\mathrm{~B}$ & 10.1 & $\mathrm{D}$ & 10.0 & c & 8.89 & C & 9.54 & $\mathrm{~A}$ & 8.71 & A & 7.44 & A & 8.35 & $\mathrm{~A}$ & 5.48 & A & 7.85 & A \\
\hline 5 & 7.10 & $C$ & 8.95 & C & 7.62 & $\mathrm{~A}$ & 14.8 & $\mathrm{D}$ & 10.5 & $A$ & 9.85 & $\mathrm{~B}$ & 9.60 & C & 10.4 & c & 8.60 & $A$ & 7.56 & A & 5.83 & A & 9.04 & $A$ \\
\hline 6 & 9.33 & A & 9.31 & $B$ & 8.87 & A & 18.2 & $\mathrm{D}$ & 8.89 & $\mathrm{~A}$ & 9.12 & A & 10.0 & C & 14.1 & $\mathrm{D}$ & 9.33 & $A$ & 9.00 & $\mathrm{~A}$ & 7.17 & A & 8.73 & A \\
\hline 7 & 8.71 & $A$ & 9.17 & A & 9.56 & $\mathrm{~A}$ & 9.06 & c & 8.98 & $A$ & 10.1 & A & 9.94 & A & 9.19 & $\mathrm{~A}$ & 9.35 & A & 8.92 & A & 15.6 & $\mathrm{D}$ & 9.35 & $A$ \\
\hline 8 & 7.94 & A & 9.21 & A & 5.19 & $\mathrm{~A}$ & 5.79 & c & 8.71 & A & 8.75 & B & 9.27 & C & 16.4 & $\mathrm{D}$ & 7.46 & $A$ & 8.44 & $\mathrm{~A}$ & 16.2 & $\mathrm{D}$ & 6.94 & $\mathrm{~A}$ \\
\hline $\bar{x}$ & \multicolumn{8}{|c|}{8.063} & \multicolumn{8}{|c|}{9.406} & \multicolumn{8}{|c|}{8.334} \\
\hline$\sigma$ & \multicolumn{8}{|c|}{1.875} & \multicolumn{8}{|c|}{0.637} & \multicolumn{8}{|c|}{0.984} \\
\hline BS & \multicolumn{8}{|c|}{$>8.1$} & \multicolumn{8}{|c|}{$>9.4$} & \multicolumn{8}{|c|}{$>8.3$} \\
\hline${ }_{(B S)}^{Q}$ & \multicolumn{8}{|c|}{ very good durability } & \multicolumn{8}{|c|}{ very good durability } & & & very $g$ & ood c & durab & & & \\
\hline $\mathrm{CV}$ & & & & 0.23 & & & & & & & & 0.06 & & & & & & & & 0.11 & & & & \\
\hline $\begin{array}{l}\mathrm{QL} \\
(\mathrm{CV})\end{array}$ & & & poor & cons & isten & & & & & & ery goc & od cc & onsist & ency & & & & & good & cons & sisten & & & \\
\hline & ability & $\operatorname{lm} \boldsymbol{R}$ & orovem & ent & BS en & hanc & emen & & & & & 16.6 & $65 \%$ & & & & & & & 3.36 & $6 \%$ & & & \\
\hline & onsist & ncy & impro & vem & ent (C & rea & uction & & & & & 71.3 & $37 \%$ & & & & & & & 49.5 & $8 \%$ & & & \\
\hline
\end{tabular}


Table 11: Binding strength (BS) and coefficient of variation values (CV), for CR office book blocks, with binding (QL $\left.L_{B S}\right)$ and consistency $\left(Q L_{C V}\right)$ quality levels. Durability and consistency improvement of nano-modified adhesives compared with a neat PVAC [\%].

\begin{tabular}{|c|c|c|c|c|c|c|c|c|c|c|c|c|c|c|c|c|c|c|c|c|c|c|c|c|}
\hline \multicolumn{25}{|c|}{ CR office $-80 \mathrm{~g} / \mathrm{m}^{2}[\mathrm{~N} / \mathrm{cm}]$} \\
\hline & \multicolumn{8}{|c|}{ PVAc (reference) } & \multicolumn{8}{|c|}{$\mathrm{PVAc}+1 \% \mathrm{SiO}_{2}$} & \multicolumn{8}{|c|}{$\mathrm{PVAc}+1 \% \mathrm{TiO}_{2}$} \\
\hline & \multicolumn{2}{|c|}{$10 \%$} & \multicolumn{2}{|c|}{$25 \%$} & \multicolumn{2}{|c|}{$50 \%$} & \multicolumn{2}{|c|}{$90 \%$} & \multicolumn{2}{|c|}{$10 \%$} & \multicolumn{2}{|c|}{$25 \%$} & \multicolumn{2}{|c|}{$50 \%$} & \multicolumn{2}{|c|}{$90 \%$} & \multicolumn{2}{|c|}{$10 \%$} & \multicolumn{2}{|c|}{$25 \%$} & \multicolumn{2}{|c|}{$50 \%$} & \multicolumn{2}{|c|}{$90 \%$} \\
\hline 1 & 8.56 & $\mathrm{C}$ & 9.46 & $\mathrm{~A}$ & 9.79 & A & 9.89 & $B$ & 9.08 & $\mathrm{~A}$ & 9.23 & A & 9.54 & A & 7.92 & $B$ & 9.54 & $B$ & 12.5 & $\mathrm{D}$ & 9.19 & A & 6.98 & $A$ \\
\hline 2 & 9.35 & A & 5.89 & A & 7.89 & A & 9.14 & C & 9.29 & $B$ & 9.64 & A & 8.42 & A & 8.02 & A & 8.10 & A & 7.96 & A & 7.62 & A & 8.12 & A \\
\hline 3 & 7.54 & A & 8.62 & A & 5.69 & $A$ & 13.3 & D & 8.64 & A & 9.27 & A & 7.54 & C & 8.92 & A & 9.52 & $B$ & 8.27 & $A$ & 8.25 & $\mathrm{~A}$ & 7.06 & A \\
\hline 4 & 4.23 & B & 6.94 & $\mathrm{C}$ & 8.00 & A & 9.83 & C & 9.48 & $A$ & 9.31 & $A$ & 9.21 & A & 7.60 & A & 9.50 & A & 8.67 & A & 8.25 & A & 6.67 & $B$ \\
\hline 5 & 5.04 & A & 4.54 & A & 7.64 & $\mathrm{C}$ & 9.37 & $\mathrm{~B}$ & 8.19 & $\mathrm{C}$ & 9.29 & $\mathrm{~B}$ & 8.67 & A & 8.98 & A & 8.21 & A & 9.29 & $A$ & 9.21 & $B$ & 6.44 & A \\
\hline 6 & 4.58 & C & 7.94 & $C$ & 8.67 & $A$ & 13.0 & $\mathrm{D}$ & 9.33 & $\mathrm{~A}$ & 9.25 & $\mathrm{~A}$ & 9.14 & $\mathrm{~A}$ & 7.98 & $B$ & 10.4 & $\mathrm{D}$ & 8.02 & $\mathrm{~A}$ & 9.04 & A & 7.37 & $B$ \\
\hline 7 & 6.52 & $\mathrm{C}$ & 9.71 & $C$ & 6.98 & $B$ & 8.29 & $\mathrm{C}$ & 8.54 & $\mathrm{~A}$ & 9.42 & $\mathrm{~A}$ & 7.17 & $\mathrm{~A}$ & 8.77 & $A$ & 8.52 & $A$ & 8.12 & $\mathrm{~A}$ & 7.06 & $\mathrm{~A}$ & 6.50 & $B$ \\
\hline 8 & 6.67 & $B$ & 8.00 & $\mathrm{C}$ & 7.80 & $B$ & 9.00 & $B$ & 9.87 & $C$ & 9.35 & $\mathrm{~B}$ & 8.10 & A & 8.54 & $B$ & 7.73 & A & 9.29 & $\mathrm{~A}$ & 7.87 & $\mathrm{~A}$ & 8.75 & $\mathrm{~A}$ \\
\hline $\bar{x}$ & \multicolumn{8}{|c|}{7.720} & \multicolumn{8}{|c|}{8.804} & \multicolumn{8}{|c|}{8.171} \\
\hline$\sigma$ & \multicolumn{8}{|c|}{1.737} & \multicolumn{8}{|c|}{0.627} & \multicolumn{8}{|c|}{0.773} \\
\hline BS & \multicolumn{8}{|c|}{$>7.7$} & \multicolumn{8}{|c|}{$>8.8$} & \multicolumn{8}{|c|}{$>8.2$} \\
\hline $\begin{array}{c}\mathrm{QL} \\
\text { (BS) }\end{array}$ & \multicolumn{8}{|c|}{ very good durability } & \multicolumn{8}{|c|}{ very good durability } & & & very $g$ & ood & durab & & & \\
\hline CV & & & & 0.2 & & & & & & & & 0.07 & & & & & & & & 0.0 & & & & \\
\hline $\begin{array}{l}\mathrm{QL} \\
(\mathrm{CV})\end{array}$ & & & poor & con & sisten & & & & & & very go & $\mathrm{od} \mathrm{cc}$ & onsist & ency & & & & & ery go & $o d c$ & onsist & ency & & \\
\hline & rability & 筮 & orovem & 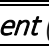 & BS en & 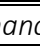 & emen & & & & & 14.0 & $3 \%$ & & & & & & & 5.8 & $4 \%$ & & & \\
\hline & onsist & $n c y$ & impro & vem & ent $(C$ & rec & uction & & & & & 69.2 & $7 \%$ & & & & & & & 58.9 & $7 \%$ & & & \\
\hline
\end{tabular}

\subsection{Book opening behaviour}

Book blocks may show large differences in their opening behaviour, which mainly depends upon the nature of adhesive, binding technique and used paper. Figures $1-5$ show differences in opening behaviour for book blocks made of the same paper type, but with different adhesive applied. Book block opening place was the same for all book blocks, at $75 \%$ of total number of pages - between page 74 and 75 .
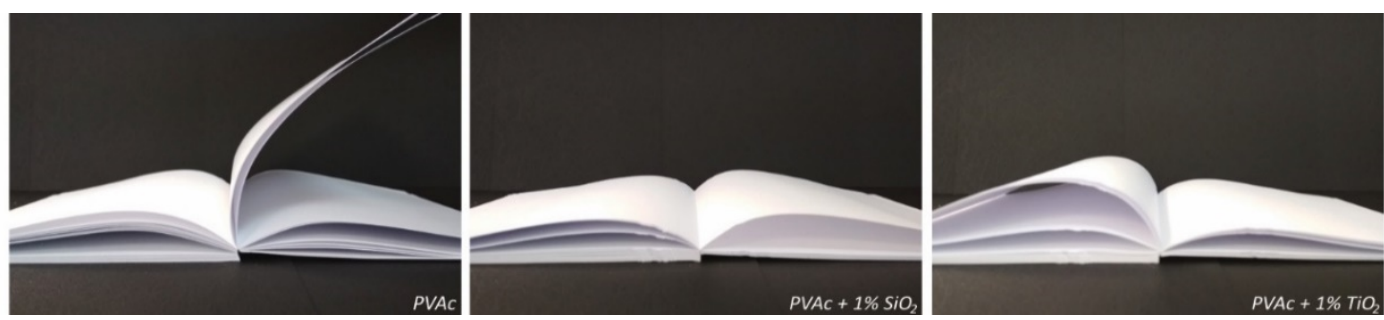

Figure 1: Opening behaviour of WFU book blocks (PVAc; PVAc $\left.+1 \% \mathrm{SiO}_{2} ; \mathrm{PVAc}+1 \% \mathrm{TiO}_{2}\right)$
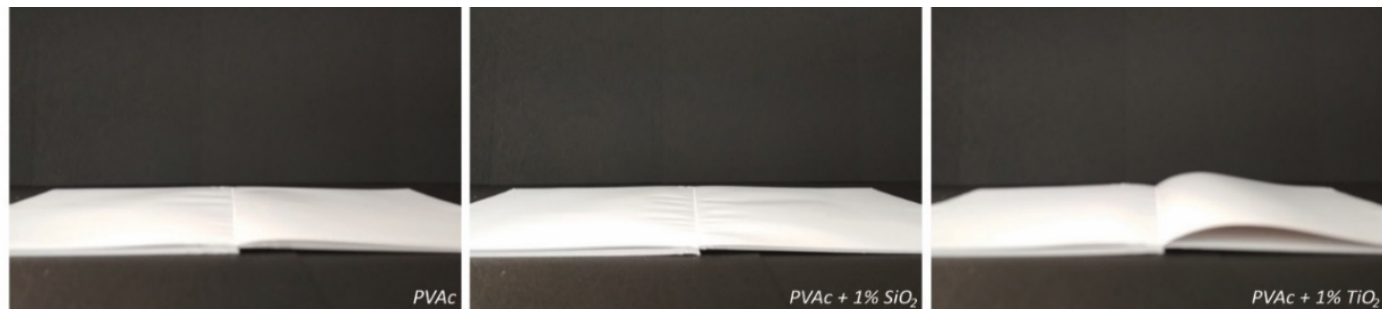

Figure 2: Opening behaviour of WFC book blocks (PVAc; PVAc + 1\% $\left.\mathrm{SiO}_{2} ; \mathrm{PVAc}+1 \% \mathrm{TiO}_{2}\right)$
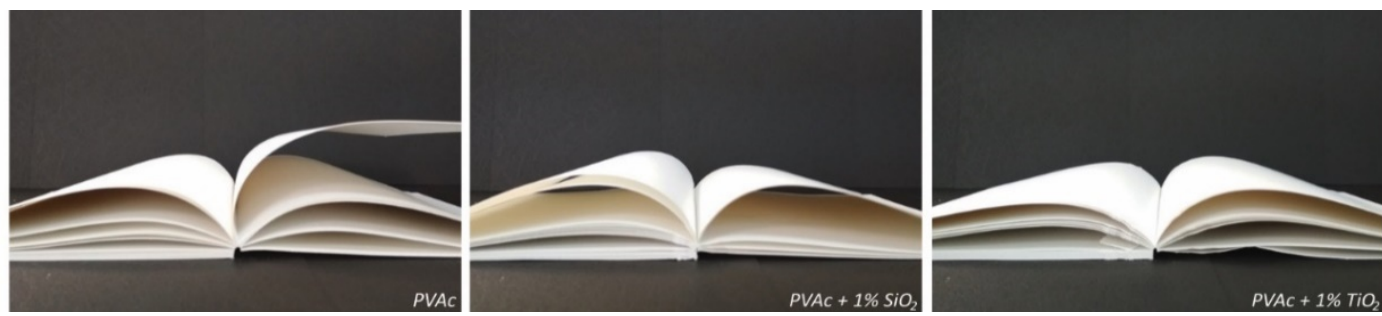

Figure 3: Opening behaviour of CW book blocks (PVAc; PVAc $\left.+1 \% \mathrm{SiO}_{2} ; \mathrm{PVAC}+1 \% \mathrm{TiO}_{2}\right)$ 

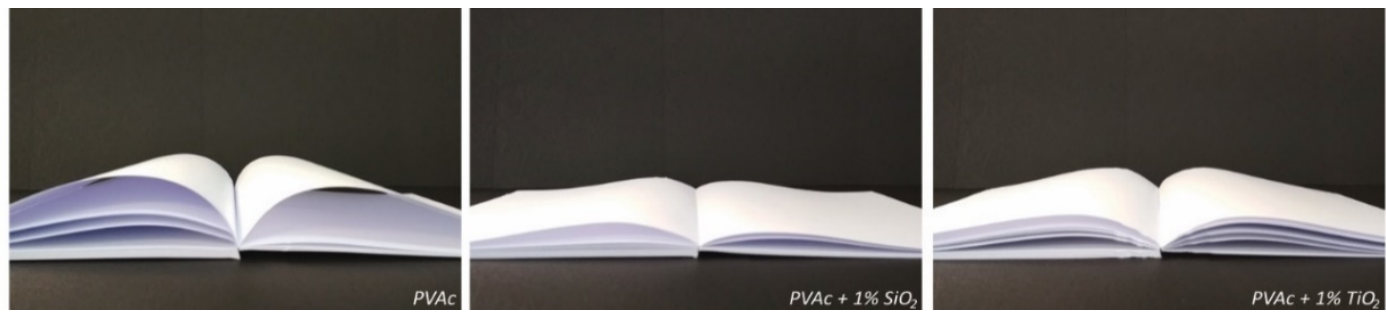

Figure 4: Opening behaviour of WF office book blocks (PVAc; PVAc $\left.+1 \% \mathrm{SiO}_{2} ; \mathrm{PVAc}+1 \% \mathrm{TiO}_{2}\right)$
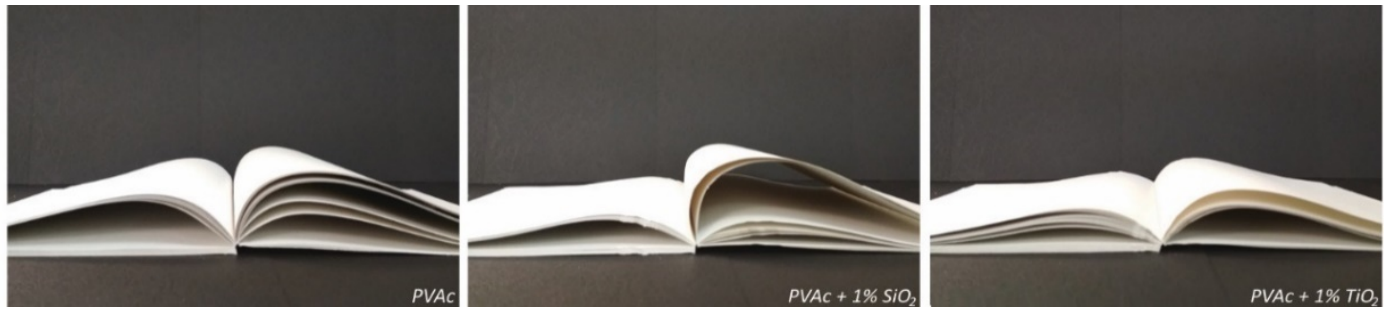

Figure 5: Opening behaviour of CR office book blocks (PVAc; $P V A c+1 \% \mathrm{SiO}_{2} ; \mathrm{PVAc}+1 \% \mathrm{TiO}_{2}$ )

Paper drape factor values for machine direction (MD) of all used papers are listed in Table 12.

Table 12: Paper drape factor values

\begin{tabular}{c|ccccc}
\hline PAPER & WFU & WFC & CW & WF office & CR office \\
\hline DRAPE FACTOR (MD) & 33 & 30 & 29 & 27 & 25 \\
\hline
\end{tabular}

\section{DISCUSSION}

According to the page-pull binding strength results (as quality measure of the binding quality) durability of 12 out of 15 groups of book blocks was classified as very good (>9,8 N/cm to $>7.7 \mathrm{~N} / \mathrm{cm})$. Other 3 groups (WFC paper) have poor or sufficient durability $(3.7 \mathrm{~N} / \mathrm{cm}$ to $5.1 \mathrm{~N} / \mathrm{cm})$. Due to the fact that paper sets the criteria for a book's structure (Jermann, 2004), all together with adhesive and paper-adhesive compatibility the criteria for a binding quality, it is necessary to observe how paper type can affect the binding quality. Thus, three different characteristics must be observed: paper bindability (binding strength), paper strength (tensile index) and paper stiffness (drape factor).

Maximum binding strength was achieved for CW book blocks (> 9.8 to $>8.6 \mathrm{~N} / \mathrm{cm}$ ), followed by WFU $(>9.6$ to $>8.4 \mathrm{~N} / \mathrm{cm})$, WF office $(>9.4$ to $>8.1 \mathrm{~N} / \mathrm{cm})$ and $C R$ office $(>8.8$ to $>7.7 \mathrm{~N} / \mathrm{cm}$ ) book blocks. The poorest binding strength was achieved for WFC book blocks (3.7 to $5.1 \mathrm{~N} / \mathrm{cm}$ ) (Figure 6). The binding strength order was the same for all used adhesives. Considering the paper type, obtained order of results was expected. CW paper is the most suitable for adhesive binding since it is uncoated and it has rough surface, high tearing resistance and limited stiffness (Pasanec Preprotić, 2012). WFU and WF office papers are also suitable for adhesive binding, but they have smoother surface with more fillers and binders, which can affect the binding strength (Džogaz, 2007). CR office paper is unpredictable due to the presence of different impurities and it can be unsuitable for adhesive binding or any binding at all (Lozo, 2004; Petković at al, 2017). WFC paper is not suitable for adhesive binding. It has high paper stiffnes and smooth, coated surface, which covers cellulose fibres (Džogaz, 2007). This facts can also be confirmed with state of tested sheets (A-D), as presented in Tables 7-11. During page-pull test procedure, it was important to visually evaluate state of tested sheets, because there are different types of adhesive joint failures - adhesive and surface failure. In the case of adhesive or cohesive adhesive failure, state of tested sheet is marked with " $A$ ". In the case of surface or internal surface failure, state of tested sheet is marked with " $B$ " or " $C$ ". In case of significant glue penetration, it is not possible to pull out tested sheet and this failure is marked with "D". Most " $D$ "states were recorded for CW book blocks, due to high surface roughness. " $A$ " states were the most recorded for all book blocks. Remarkable number of "B" and " $C$ " states was recorded for CR book blocks that can be linked to paper type, unpredictable size and share of recycled cellulose fibres and impurities.

Machine direction tensile index was quite similar for WFU, CW and CR paper (41.15 to $47.09 \mathrm{Nm} / \mathrm{g}$, respectively). WF office paper showed the highest tensile index $-76.03 \mathrm{Nm} / \mathrm{g}$, while WFC showed the 
lowest value $-31.66 \mathrm{Nm} / \mathrm{g}$. Considering the obtained results for binding strength, paper strength can be directly linked to binding strength quality. However, adhesive-paper compatibility remained the main factor for binding strength (Figure 6).

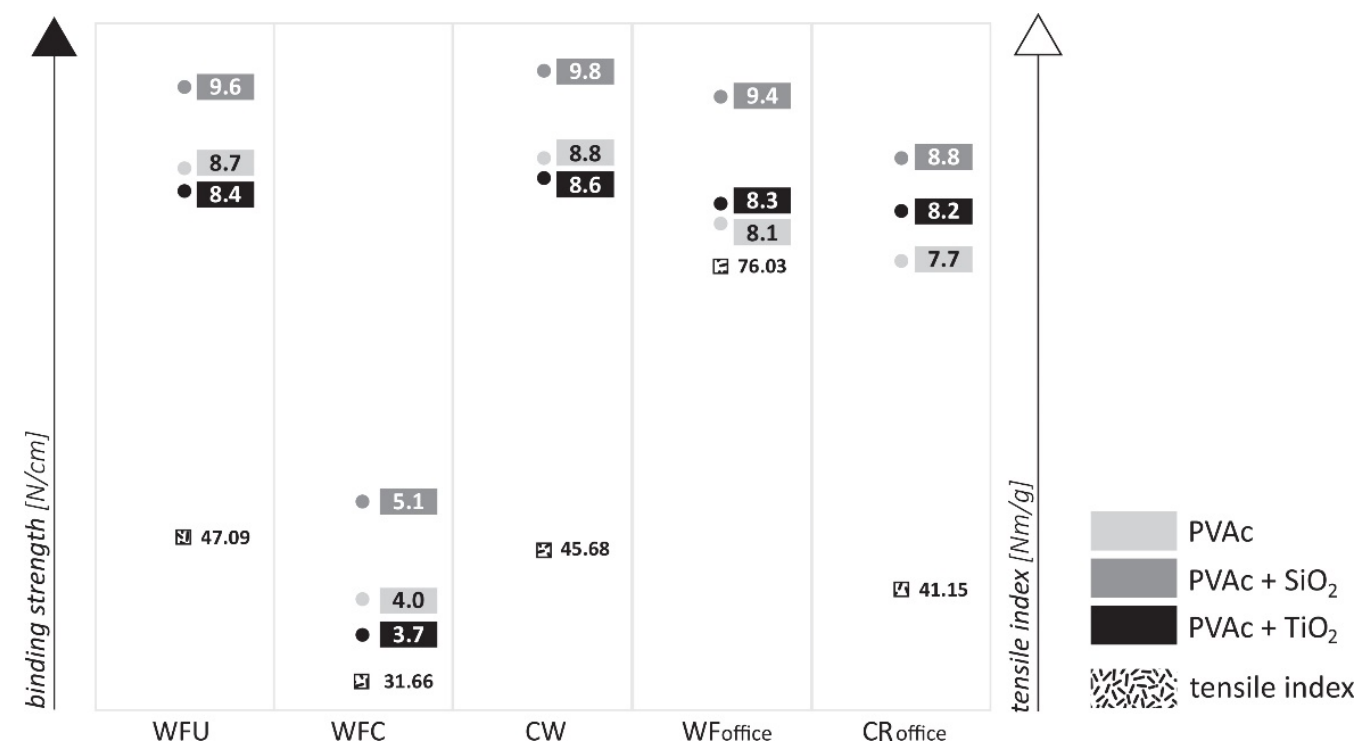

Figure 6: Comparison of binding strength (marked with circle) for different paper type book blocks and importance of tensile index (marked with square)

The aim of this study was the improvement of the PVAc adhesive binding performance by nanoparticles modification, namely by $1 \%$ of $\mathrm{SiO}_{2}$ and $\mathrm{TiO}_{2}$ nanoparticles. Enhancement of binding strength was achieved by $1 \%$ of $\mathrm{SiO}_{2}$ nanoparticles, for all types of book blocks (26.64 - 10.30\%). By adding $\mathrm{TiO}_{2}$ nanoparticles, binding strength remained almost unchanged (-2.68 - 5.84\%). In addition, another important feature was the reduction of coefficient of variation by adding $\mathrm{SiO}_{2}$ nanoparticles (43.28-71.37\%), as the main factor of the binding quality consistency.

It was also necessary to observe differences between binding strength results considering the test position - front, middle or rear. For WFU, CW and WF office papers, which are suitable for adhesive binding, binding strength values for neat PVAc were larger at front and rear part of the book block then in the middle. For PVAc adhesive with $1 \%$ of $\mathrm{SiO}_{2}$ nanoparticles, results were uniform. The increase in uniformity for PVAc adhesive with $1 \%$ of $\mathrm{TiO}_{2}$ nanoparticles was not important, since considerably higher binding strength for $\mathrm{TiO}_{2}$ adhesive modification was not achieved.

The best binding performance of adhesive depends upon the binding strength, but also upon the visual and tactical experiences of end user. For good end user experience, book opening behaviour should have satisfactory characteristics, including lay-flat, easy-open flexible spine and adequate spine opening. Different adhesive - paper combinations show large differences in book opening behaviour. Papers with higher drape factor are stiffer and they tend to remain rigidly upright when book is open or they require a force in order to get fully opening of a book into a readable position. When book is fully open, the joint between leaves is subjected to peeling forces, which can be controlled by motion of the spine (Bracić, 2017; Jermann, 2004). In a fully flat opening, the most user-friendly type of opening, it is hard to achieve durable book blocks, especially with coated papers (Figure 2). In addition, a surface adhesion failure can occur between the paper and its coatings when applying elastic adhesives that allow movement, such as PVAc. This was confirmed with a poor to sufficient binding strength results and poor consistency for WFC book blocks. Completely opposite to a flat opening, is fixed spine which prevents any movement and users have to hold the book open (Figure 1 and 3; PVAc). This can be explained by higher drape factor of WFU and CW papers compared to other papers in this research. However, the possibility of durable book blocks with controlled spine opening (Figure 1 and 3; PVAc $+1 \% \mathrm{SiO}_{2} ; \mathrm{PVAc}+1 \% \mathrm{TiO}_{2}$ ) production was achieved by using nano-modified adhesive (especially PVAC $+1 \% \mathrm{SiO}_{2}$ ). Controlled spine opening was also achieved for book blocks made of WF and CR office papers with neat PVAc and PVAc modified with $1 \% \mathrm{TiO}_{2}$ (Figure 4 and 5; PVAc; PVAc $+1 \% \mathrm{TiO}_{2}$ ). WF office paper in combination with $\mathrm{PVAc}+1 \% \mathrm{SiO}_{2}$ showed very good binding quality, according to its binding strength results. In addition, it can be classified as book block with the best binding performance in this research (Figure 4; PVAc $+1 \% \mathrm{SiO}$ ) due to a flat spine opening book 
blocks. Opening behaviour of CR book blocks was controlled, but uneven and slack (Figure 5; PVAc $+1 \%$ $\mathrm{SiO}_{2}$ ). As it was already mentioned, unpredictable properties of recycled paper made it impossible to give a correct conclusions and predictions for opening behaviour and binding strength, e.g. binding performance of CR office book blocks.

\section{CONCLUSIONS}

The right binding type selection usually depends on the book function, content, number of pages, printing budget and target group. However, reduction of printed book volumes and delivery time leads to the increase in popularity of adhesive bonded books. According to paper-adhesive compatibility, selection of paper and adhesive should be done before the production process begins. The aim of the research was to improve the binding performance of PVAc adhesive, by its modification with $\mathrm{SiO}_{2}$ and $\mathrm{TiO}_{2}$ nanoparticle, which reflected the binding strength and book opening behaviour. Considering the obtained results of binding strength and visually evaluation of book opening behaviour, WFC paper is not suitable for doublefan adhesive binding with PVAc adhesive at all. All other used papers are suitable and in addition, they showed very good durability. By adding $\mathrm{SiO}_{2}$ nanoparticles, the binding strength increased by 10.30 $16.65 \%$ respectively, for all groups of papers suitable for binding (WFU, CW, WF and CR office). Notable increase or decrease in binding strength with $\mathrm{TiO}_{2}$ nanoparticles was not recorded. In addition, book blocks bound with PVAc modified with $1 \% \mathrm{SiO}_{2}$ adhesive showed more uniform adhesive application through all test positions - front, middle and rear part of the book block. Reduction of coefficient of variations was achieved for both adhesives modified with nanoparticles $\left(43.28-71.37 \% \mathrm{SiO}_{2} ; 4.26-58.97 \% \mathrm{TiO}_{2}\right.$ ), which can guarantee better consistency of binding quality. Despite the end product page-pull test results for the evaluation of binding performance, visual evaluation of book block opening behaviour should be done as well. The best easy-open, lay-flat opening behaviour was achieved for combination of WF office paper and $\mathrm{PVAc}+1 \% \mathrm{SiO}_{2}$ adhesive. By adding $\mathrm{SiO}_{2}$ nanoparticles in PVAc adhesive, book blocks made of stiff papers (WFU, CW) had more openable book behaviour. Although, the price of PVAc adhesive with $1 \% \mathrm{SiO}_{2}$ nanoparticles increased, obtained advantages are of particular importance for the end product quality. During nano-modified adhesive preparation, it is of great importance to achieve good distribution of nano particles, in order to avoid particle aggregation. In addition, it is important to find an optimum content of nano particles, because at higher contents the properties may deteriorate.

\section{ACKNOWLEDGMENTS}

The authors are grateful for the support of the University of Zagreb, Grant under the title: "Modifikacije konvencionalnih grafičkih materijala nanočesticama i kromogenim materijalima i njihova zdravstvena ispravnost" ("Modification of conventional graphic materials with nanoparticles and chromogenic materials and their health safety").

\section{REFERENCES}

[1] Bardak, T., Tankut, A.N., Tankut, N., Sozen, E., Aydemir, D.: "The effect of nano-TiO2 and SiO2 on bonding strength and structural properties of poly (vinyl acetate) composites", Measurement 93, 80-85, 2016. doi: 10.1016/j.measurement.2016.07.004.

[2] Bonnefond, A., Reyes, Y., Peruzzo, P., Ronne, E., Fare, J., Paulis, M., Leiza, J.R.: "Effect of the Incorporation of Modified Silicas on the Final Properties of Wood Adhesives", Macromolecular Reaction Engineering 7(10), 527-537, 2013. doi: 10.1002/mren.201300117.

[3] Bracić, M.: “Utjecaj krutosti papira na kvalitetu bešavne forme s PUR ljepilom”, MSc thesis, University of Zagreb, 2017.

[4] Džogaz, N.: "Ispitivanje kvaliteta knjiga povezanih na mašini za lepljeni povez Horizon BQ-270", BSc thesis, University of Novi Sad, 2007.

[5] Ebnesajjad, S.: "Adhesives Technology Handbook", $2^{\text {nd }}$ ed, (Wiliam Andrew, Norwich, USA, 2008), page 114 .

[6] Intergraf: "2015 Intergraf European Book Market Report", European Federation for Print and Digital Communication, 2015.

[7] Intergraf: "2017 Intergraf European Economic Report - The evolution of the European graphic industry", European Federation for Print and Digital Communication, 2017. 
[8] Jermann, P.: "Reflections on Book Structures - part 2", Temper Productions, 2004, URL: http://temperproductions.com/Bookbinding\%20How-to/Reflections/reflect2.htm (last request: 2018-05-06).

[9] Jermann, P.: "Reflections on Book Structures - part 3", Temper Productions, 2008, URL: http://temperproductions.com/Bookbinding\%20How-to/Reflections/Reflections\%203\%20\%20Spine\%20Control.pdf (last request: 2018-06-04).

[10] Jović, M., Buhin, Z., Krobot, I., Lučić Blagojević, S.: "Analysis of the Environmental Sustainability of the Adhesive Technology", Kemija u industriji 60(5), 269-276, 2011.

[11] Laskowska, A., Kozakiewicz, P.: "Surface Wettability of Wood Species from Tropical and Temperate Zones by Polar and Dispersive Liquids", Drvna industrija 68(4), 299-306, 2017. doi: 10.5552/drind.2017.1704.

[12] Lozo, B.: "Doprinosi optimiranju kvalitete novinskog papira", MSc thesis, University of Zagreb, 2004.

[13] Packham, D.E.: "Handbook of Adhesion", $2^{\text {nd }}$ ed, (John Wiley \& Sons, Chichester, England, 2005), pages 136-138. doi: 10.1002/0470014229.

[14] Paris, J.: "Adhesives for paper, board and foils", International Journal of Adhesion \& Adhesives 20(2), 89-90, 2000.

[15] Pasanec Preprotić, S.: "Čvrstoća knjižnog bloka u ovisnosti o starenju”, PhD thesis, University of Zagreb, 2012.

[16] Petković, G., Rožić, M., Vukoje, M., Pasanec Preprotić, S.: "Interactions in polyvinyl acetate - paper adhesive joint and influence on its adhesion parameters", Proceedings of International Symposium on Graphic Engineering and Design 2016, (University of Novi Sad, Novi Sad, Srbija, 2016), pages 91-101.

[17] Petković, G., Pasanec Preprotić, S., Banić, D.: "Evaluation of finished product quality depending on paper properties and binding technique", Polytechnic and Design 5(3), 237-247, 2017. doi: 10.19279/TVZ.PD.2017-5-3-19.

[18] Petrie, E.M., Bookbinding Adhesives, ASI Adhesives \& Sealants Industry, 2008, URL: https://www.adhesivesmag.com/articles/87133-bookbinding-adhesives (last request: 2018-09-05).

[19] Ramljak, A.: “Adhezijska svojstva polivinil acetatnog ljepila”, BSc thesis, University of Zagreb, 2017.

[20] Rebsamen, W., Adhesive Binding Library Books, The New library scene, 1983, URL: http://www.mekatronicsinc.com/machines/Werner_1983.pdf (last request: 2018-09-05)

[21] Salvini, A., Saija, L.M., Finocchiaro, S., Gianni, G., Giannelli, C., Tondi, G.: "A New Methodology in the Study of PVAc-Based Adhesive Formulations", Journal of Applied Polymer Science 114(6), 3841-3854, 2009. doi: 10.1002/app.31032.

[22] Šedivka, P., Bomba, J., Böhm, M., Boška, P.: "Influence of Temperature on the Strength of Bonded Joints", BioResources 10(3), 3999-4010, 2015.

[23] Thomson, P.: "Bookbinding Tutorial: Glues - Tips, Techniques, Types \& Recipes", ibookbinding, 2014, URL: https://www.ibookbinding.com/blog/bookbinding-gluing-tips-techniques-types-info/ (last request: 2018-08-30).

[24] Wen, N., Tang, Q., Chen, M., Wu, L.: "Synthesis of PVAc/SiO2 latices stabilized by silica nanoparticles", Journal of Colloid and Interface Science 320(1), 152-158, 2008. doi: 10.1016/j.jcis.2007.11.059.

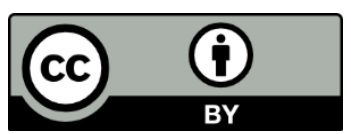

(C) 2018 Authors. Published by the University of Novi Sad, Faculty of Technical Sciences, Department of Graphic Engineering and Design. This article is an open access article distributed under the terms and conditions of the Creative Commons Attribution license 3.0 Serbia (http://creativecommons.org/licenses/by/3.0/rs/). 